\title{
Regulation of Intestinal UDP-Glucuronosyltransferase 1 A1 by the Farnesoid X Receptor Agonist Obeticholic Acid Is Controlled by Constitutive Androstane Receptor through Intestinal Maturation ${ }^{\sqrt{\mathrm{S}}}$
}

\author{
André A. Weber, Elvira Mennillo, Xiaojing Yang, Lori W.E. van der Schoor, Johan W. Jonker, \\ Shujuan Chen, and Robert H. Tukey
}

Laboratory of Environmental Toxicology, Department of Pharmacology, University of California, San Diego, La Jolla, California (A.A.W., E.M., X.Y., S.C., R.H.T.) and Department of Pediatrics, Center for Liver, Digestive and Metabolic Diseases, University of Groningen, University Medical Center Groningen, Groningen, The Netherlands (L.W.E.v.d.S., J.W.J.)

Received September 4, 2020; accepted October 14, 2020

\begin{abstract}
UDP-glucuronosyltransferase (UGT) $1 \mathrm{~A} 1$ is the only transferase capable of conjugating serum bilirubin. However, temporal delay in the development of the UGT1A1 gene leads to an accumulation of serum bilirubin in newborn children. Neonatal humanized UGT1 (hUGT1) mice, which accumulate severe levels of total serum bilirubin (TSB), were treated by oral gavage with obeticholic acid (OCA), a potent FXR agonist. OCA treatment led to dramatic reduction in TSB levels. Analysis of UGT1A1 expression confirmed that OCA induced intestinal and not hepatic UGT1A1. Interestingly, Cyp2b10, a target gene of the nuclear receptor CAR, was also induced by OCA in intestinal tissue. In neonatal hUGT1/Car ${ }^{-1-}$ mice, OCA was unable to induce CYP2B10 and UGT1A1, confirming that CAR and not FXR is involved in the induction of intestinal UGT1A1. However, OCA did induce FXR target genes, such as Shp, in both intestines and liver with induction of Fgf15 in intestinal tissue. Circulating FGF15 activates hepatic FXR and, together with hepatic Shp, blocks Cyp7a1 and Cyp7b1 gene expression, key enzymes in bile acid metabolism. Importantly, the administration of OCA in neonatal hUGT1 mice accelerates intestinal epithelial cell maturation, which
\end{abstract}

directly impacts on induction of the UGT1A1 gene and the reduction in TSB levels. Accelerated intestinal maturation is directly controlled by CAR, since induction of enterocyte marker genes sucrase-isomaltase, alkaline phosphatase 3 , and keratin 20 by OCA does not occur in hUGT1/Car ${ }^{-1-}$ mice. Thus, new findings link an important role for CAR in intestinal UGT1A1 induction and its role in the intestinal maturation pathway.

\section{SIGNIFICANCE STATEMENT}

Obeticholic acid (OCA) activates FXR target genes in both liver and intestinal tissues while inducing intestinal UGT1A1, which leads to the elimination of serum bilirubin in humanized UGT1 mice. However, the induction of intestinal UGT1A1 and the elimination of bilirubin by OCA is driven entirely by activation of intestinal CAR and not FXR. The elimination of serum bilirubin is based on a CARdependent mechanism that facilitates the acceleration of intestinal epithelium cell differentiation, an event that underlies the induction of intestinal UGT1A1.
Introduction

Humanized UGT1 ( $h U G T 1$ ) mice express the entire human UGT1 locus (9-UGT1 genes) in a $U g t 1$-null background and have served as an excellent animal model to examine the underlying processes linking important developmental events with gene expression (Fujiwara et al., 2010). One of the more significant events is the delayed developmental expression of the UGT1Al gene, which is solely responsible for the metabolism of serum bilirubin (Fujiwara et al., 2012). Like the hepatic expression patterns in newborns, the UGTIAl gene is developmentally

This work was supported by National Institutes of Health Grants [ES010337] and [GM126074] (to R.H.T.) and [R21-Al135677] (to S.C.).

https://doi.org/10.1124/dmd.120.000240.

S This article has supplemental material available at dmd.aspetjournals.org. delayed in newborn neonatal mice, resulting in the accumulation of severe levels of serum bilirubin. In humans, most cases of hyperbilirubinemia in children are benign, except when the rapid onset of severe neonatal hyperbilirubinemia (SNH) is not monitored or prevented (Chen and Tukey, 2018). Major risk factors that lead to the onset of SNH include accelerated hemolysis brought on by rhesus disease and ABO incompatibility, glucose-6-phosphate dehydrogenase deficiency, infections, breastfeeding, and premature birth (Bhutani et al., 2013; Maisels, 2015; Olusanya et al., 2015; Wong and Stevenson, 2015; Cunningham et al., 2016). Extreme levels of total serum bilirubin (TSB) can lead to early or acute bilirubin encephalopathy, which presents early as lethargy and poor feeding but can progress to hypo- and hypertonia, high-pitched crying, muscle spasms, opisthotonos, seizures, and even death (Bhutani and Johnson-Hamerman, 2015). The more chronic form, which proceeds acute bilirubin encephalopathy, is termed kernicterus (Kaplan et al., 2011).

ABBREVIATIONS: AhR, aryl hydrocarbon receptor; Akp3, alkaline phosphatase 3; BisTris, 2-[bis(2-hydroxyethyl)amino]-2-(hydroxymethyl) propane-1,3-diol; CAR, constitutive androstane receptor; DAPT, $N$-[N-(3,5-difluorophenacetyl)-L-alanyl]-S-phenylglycine t-butyl ester; DPBS, Dulbecco's phosphate-buffered saline; FGF, fibroblast growth factor; FXR, farnesoid X receptor; HRP, horseradish peroxidase; hUGT1, humanized UGT1; IEC, intestinal epithelium cell; Krt20, keratin 20; LXR, liver X receptor; NCoR1, nuclear receptor corepressor 1; Nox4, NADPH oxidase 4; OCA, obeticholic acid; PPAR $\alpha$, peroxisome proliferator-activated receptor $\alpha$; PXR, pregnane X receptor; Shp, small heterodimer partner; SI, small intestine; Sis, sucrase-isomaltase; SNH, severe neonatal hyperbilirubinemia; TSB, total serum bilirubin; UGT, UDP-glucuronosyltransferase. 
TABLE 1

Primer sequences used for reverse-transcription quantitative polymerase chain reaction (RT-qPCR)

\begin{tabular}{|c|c|c|}
\hline Primer & Forward & Reverse \\
\hline Human UGTIAI & 5'-ССТTGCCTCATCAGAATTCCTTC-3' & 5'-ATTGATCCCAAAGAGAAAACCAC-3' \\
\hline Mouse Cyp $2 b 10$ & 5'-AAAGTCCCGTGGCAACTTCC-3' & 5'-CATCCCAAAGTCTCTCATGG-3' \\
\hline Mouse Nqol & 5'-TTTAGGGTCGTCTTGGCAAC-3' & 5'-GTCTTCTCTGAATGGGCCAG-3' \\
\hline Mouse Cyp 4 a10 & 5'-CACACCCTGATCACCAACAG-3' & 5'-TCCTTGATGCACATTGTGGT-3' \\
\hline Mouse Cyp3a11 & $5^{\prime}$-ACAAACAAGCAGGGATGGAC-3' & 5'-CCCATATCGGTAGAGGAGCA-3' \\
\hline Mouse Cyclophiline & 5'-CAGACGCCACTGTCGCTT-3' & 5'-TGTCTTTGGAACTTTGTCTGC-3' \\
\hline Mouse Shp & 5'-CACGATCCTCTTCAACCCAG-3' & 5'-AAGACTTCACACAGTGCCCA-3' \\
\hline Mouse Cyp $8 b 1$ & 5'-GATAGGGGAAGAGAGCCACC-3' & 5'-TCCTCAGGGTGGTACAGGAG-3' \\
\hline Mouse Cyp $27 a 1$ & 5'-GGGCACTAGCCAGATTCACA-3' & 5'-CTATGTGCTGCACTTGCCC-3' \\
\hline Mouse Cyp7b1 & 5'-TTATCAAGGGTGGTTCACGA-3' & 5'-TCCTAGGCCTTCTCTTTGCC-3' \\
\hline Mouse $A k p 3$ & 5'-CTGGAGCCCTACACCGACT-3' & 5'-AGGCTTCTGGCGCTGTTAT-3' \\
\hline Mouse Sis & 5'-ACCCCTAGTCCTGGAAGGTG-3' & 5'-CACATTTTGCCTTTGTTGGATGC-3' \\
\hline Mouse $\mathrm{Krt20}$ & 5'-CCTGCGAATTGACAATGCTA-3' & $5^{\prime}$-CCTTGGAGATCAGCTTCCAC-3' \\
\hline Mouse Glb1 & 5'-CACTGCTGCAACTGCTGG-3' & 5'-ATGTATCGGAATGGCTGTCC-3' \\
\hline
\end{tabular}

Glb1, galactosidase $\beta 1$; Lrp2, low-density lipoprotein-related protein 2.

In approximately $10 \%$ of newborn $h U G T 1$ mice, SNH develops and leads to spontaneous seizures and death (Chen and Tukey, 2018). One of the central nervous system patterns that develops in these mice is a dramatic reduction in nerve myelination, an event that we had speculated contributes to the seizure pattern (Barateiro et al., 2016). The human UGT1A1 gene is regulated by events that lead to activation of the pregnane X receptor (PXR) (Chen et al., 2012), constitutive androstane receptor (CAR) (Yoda et al., 2017; Paszek and Tukey, 2020), and the peroxisome proliferator-activated receptor $\alpha(\operatorname{PPAR} \alpha)$ (Senekeo-Effenberger et al., 2007), followed by induction of the $U G T 1 A 1$ gene in either the liver or intestines. Induction of UGTIAl in neonatal $h U G T 1$ mice results in a precipitous drop in serum bilirubin levels, thus serving as an excellent animal model to screen and test therapeutics that could be used to treat infants with SNH (Chen and Tukey, 2018). The challenge is to identify agents that do not share toxicities when administered to humans.

An exciting class of drugs that are being investigated for a number of liver and biliary abnormalities are selective farnesoid $\mathrm{X}$ receptor (FXR) agonists such as obeticholic acid (OCA). Obeticholic acid has been studied in conditions such as primary biliary cholangitis with beneficial effects while also displaying a capacity to reduce TSB levels (Parés et al., 2020). Although OCA is an effective agonist for the activation of FXR, there is no evidence that activated FXR targets the UGT1A1 gene. To examine this possibility in greater detail, experiments have been conducted in neonatal $h U G T 1$ mice treated orally with OCA, with the surprising result that treatment dramatically lowers TSB levels. However, our findings clearly indicate that induction of UGT1A1 by OCA is not a result of FXR targeting the UGT1Al gene.

\section{Material and Methods}

Chemical Reagents. OCA was obtained from MedChemExpress (South Brunswick, NJ), and DAPT was obtained from Sigma-Aldrich (Saint Louis, MO). ORA-Plus oral suspension solution was from Perrigo (Allegan, MI). Antibodies used for Western blotting were as follows: anti-UGT1A1 (Ab-170858) and antiCYP7A1 (Ab-65596) from Abcam; anti-GAPDH (sc-32233), anti-FGF15 (sc514647), and anti-SIS (sc-27603) from Santa Cruz Technologies; anti-CYP2B10 (a kind gift from Dr. Masahiko Negishi, National Institute of Environmental Health Sciences; anti-mouse IgG horseradish peroxidase (HRP)-conjugated antibody and anti-rabbit IgG HRP-conjugated antibodies were obtained from Cell Signaling Technology, Inc. (Danvers, MA); and anti-goat IgG HRPconjugated antibody was from Jackson Immunoresearch (West Grove, PA).
Animals. Transgenic mice expressing the human $U G T 1$ locus in a $U g t 1^{-1-}$ background ( $h U G T 1)$ were developed previously (Fujiwara et al., 2010). The Car-null $\left(\mathrm{Car}^{-1-}\right)$ mice were a gift from Dr. Masahiko Negishi (National Institute of Environmental Health Sciences) and were crossed with $h U G T 1$ mice to generate $h U G T 1 / \mathrm{Car}^{-1-}$ mice (Fujiwara et al., 2012). All animals were housed at the University of California San Diego Animal Care Facility and received food and water ad libitum. The protocols for mouse handling and all procedures were approved by the University of California San Diego Animal Care and Use Committee, and these protocols were conducted in accordance with federal regulations. Neonatal 10-day-old $h U G T 1$ or $h U G T 1 / \mathrm{Car}^{-1-}$ mice were treated by oral gavage with ORA-Plus (vehicle) or $50 \mathrm{mg} / \mathrm{kg}$ per day of OCA divided into two doses per day ( $25 \mathrm{mg} / \mathrm{kg}$ each). After 5 consecutive days, neonatal mice ( $n=3$ to 4 per group) were sacrificed, and blood, livers, and small intestines were collected.

Bilirubin Measurements. Blood from neonates was obtained from the submandibular vein, collected into an Eppendorf tube, and centrifuged at $14,000 \mathrm{~g}$ for 2 minutes. A small sample of serum was used to measure TSB levels using a Unistat Bilirubinometer (Reichert, Inc., Depew, NY).

Isolation of Crypt Cells and Organoid Culture. Intestinal crypt cell isolation and organoid culturing were carried out according to previous publications, with several modifications (Sato et al., 2009; Sato and Clevers, 2013). Briefly, mouse small intestine was dissected, cut longitudinally, and washed with ice-cold Dulbecco's phosphate-buffered saline (DPBS). The tissue was further dissected into small pieces and incubated in DPBS containing $2 \mathrm{mM}$ EDTA at $4{ }^{\circ} \mathrm{C}$ shaking for 30 minutes. Then, EDTA solution was washed out, and $10 \%$ fetal bovine serum in DPBS buffer was added, followed by vigorously shaking to release villi and crypt cells. The cell solution was filtered through a 70-mm cell strainer. The filtrate was centrifuged at $1000 \mathrm{~g}$ for 7 minutes at $4^{\circ} \mathrm{C}$, and the cell pellet was resuspended in DPBS. Crypt cells were counted. Approximately 1000 crypts were suspended into $50 \mu \mathrm{l}$ of ice-cold Matrigel and plated into prewarmed 24-well culture plate. At 20 minutes after the incubation at $37^{\circ} \mathrm{C}$, $0.5 \mathrm{ml}$ of complete growth medium (advanced Dulbecco's modified Eagle's medium with GlutaMax, Hepes, penicillin/streptomycin, N2 and B27 supplements with $2.5 \mathrm{mM} \mathrm{N}$-acetylcysteine, $0.1 \mathrm{mg} / \mathrm{ml}$ mouse Noggin, $0.05 \mathrm{mg} / \mathrm{ml}$ mouse Epidermal Growth Factor, and 10\% of R-Spondin1-conditioned medium) was added. The growth of organoids was monitored. Mouse Epidermal Growth Factor was added every other day. Fresh medium was added every 3 days. Cells were normally ready for passage every 4 to 5 days. Crypt cells were exposed to various concentrations of OCA $(50$ and $100 \mu \mathrm{M})$ and to DAPT $(10 \mu \mathrm{M})$, a known intestinal maturation inductor, as a positive control (Ogaki et al., 2013); on day 3 and after 24 hours, the cells were collected in triplicate for further analysis.

Reverse-Transcription Quantitative Polymerase Chain Reaction. Tissue samples were homogenized in $1 \mathrm{ml}$ of TRIzol Reagent (Thermo Fisher Scientific), and total RNA from whole tissues was isolated. Using iScript Reverse 
Transcriptase (Bio-Rad Laboratories, Hercules, CA), $1 \mu \mathrm{g}$ of total RNA was used for the generation of cDNA in a total volume of $12 \mu \mathrm{l}$, as outlined by the manufacturer. After cDNA synthesis, quantitative (real-time) polymerase chain reaction was carried out on a CFX96 qPCR system (Bio-Rad) using SsoAdvanced SYBR Green Supermix (Bio-Rad). All primers used in this study are reported in Table 1.

Western Blot Analysis. For whole-tissue analyses, minced liver tissue $(0.1 \mathrm{mg})$ was homogenized in $0.4 \mathrm{ml}$ of $1 \times$ radioimmunoprecipitation assay buffer lysis buffer (EMD Millipore, Billerica, MA) supplemented with protease and phosphatase inhibitor cocktail (Sigma-Aldrich). The samples were centrifuged at $16,000 \mathrm{~g}$ for 20 minutes at $4^{\circ} \mathrm{C}$, and the supernatants were transferred to a new tube and kept at $-80^{\circ} \mathrm{C}$ until analysis. Proteins were quantified using bovine serum albumin as standard.

Western blots were performed by using NuPAGE 4\%-12\% BisTrispolyacrylamide gels (Invitrogen) with the protocols described by the manufacturer. Protein $(30 \mu \mathrm{g})$ was subjected to electrophoresis at $170 \mathrm{~V}$ for 60 minutes and transferred at $20 \mathrm{~V}$ for 60 minutes to polyvinylidene fluoride membranes (EMD Millipore). Membranes were blocked with 5\% nonfat milk at room temperature for 1 hour and incubated with primary antibodies at $4^{\circ} \mathrm{C}$ overnight. Membranes were then washed and exposed to HRP-conjugated secondary antibodies (antimouse $\mathrm{IgG}$, anti-rabbit $\mathrm{IgG}$, and anti-goat $\mathrm{IgG}$ ) for 1 hour at room temperature. Protein was detected by the enhanced chemiluminescence Plus Western blotting detection system (Bio-Rad) and was visualized by the Bio-Rad Chemidoc Touch Imaging System.

Statistical Analyses. Data are represented as means \pm S.D. Statistical differences were determined by Student's $t$ test. $P$ values $<0.05$ were considered statistically significant, and these differences are indicated with $* P<0.05$, $* * P<$ 0.01 , and $* * * P<0.001$. Statistical analyses were performed using GraphPad 5 (San Diego, CA).

\section{Results}

Treatment of OCA in Neonatal hUGT1 Mice. Our laboratory previously reported that UGT1A1 can be transcriptionally induced by several nuclear receptors, such as PXR (Chen et al., 2012), CAR (Yoda et al., 2017; Paszek and Tukey, 2020), PPAR $\alpha$ (Senekeo-Effenberger et al., 2007), and liver X receptor (LXR) $\alpha$ (Hansmann et al., 2020); by the environmental sensors aryl hydrocarbon receptor (AhR) (Yueh et al., 2003) and nuclear factor erythroid-2-related factor 2 (Yueh and Tukey, 2007; Yoda et al., 2017); and after activation of the IkB kinase/Nuclear Factor kappa Beta pathway (Liu et al., 2016). A recent clinical report suggested that treatment with OCA, an FXR activator, lowers serum bilirubin levels (Parés et al., 2020). However, confirmation that FXR played a role in the lowering of bilirubin was not apparent, and there is no existing evidence that FXR can regulate the UGT1Al gene.

After treatment of $h U G T 1$ mice with OCA, serum bilirubin levels were reduced, indicating that UGT1A1 was induced (Fig. 1A). Analysis of gene and protein expression in the small intestine (SI) confirmed a dramatic induction of UGT1A1 (Fig. 1, B and C) only, since induction did not occur in liver tissue. This result demonstrates that intestinal UGT1A1 expression is the major regulator of bilirubin clearance in mice treated with OCA (Supplemental Fig. 1). This does not mean, however, that FXR was not activated. The induction of small heterodimer partner (Shp) gene transcripts and a decrease of $C y p 7 a 1$ transcripts and protein levels in the liver of OCA-treated neonates confirm that FXR is activated in the liver (Fig. 2A). There are two pathways involved in bile acid metabolism: the classic pathway, with the participation of CYP7A1, and the alternate (acidic) pathway, with the key enzyme CYP7B1 (Kliewer and Mangelsdorf, 2015). As demonstrated, Cyp7al gene expression is decreased in OCA-treated mice, resulting from inhibition of gene expression by FGF15. Consistent with this, Cyp $8 b 1$, a downstream enzyme in the classic pathway, is also decreased. Gene expression analysis also revealed that the alternate pathway is blocked in OCAtreated neonatal mice with the downregulation of $C y p 7 b 1$ enzyme. Only CYP27A1, which is an enzyme that participates in both
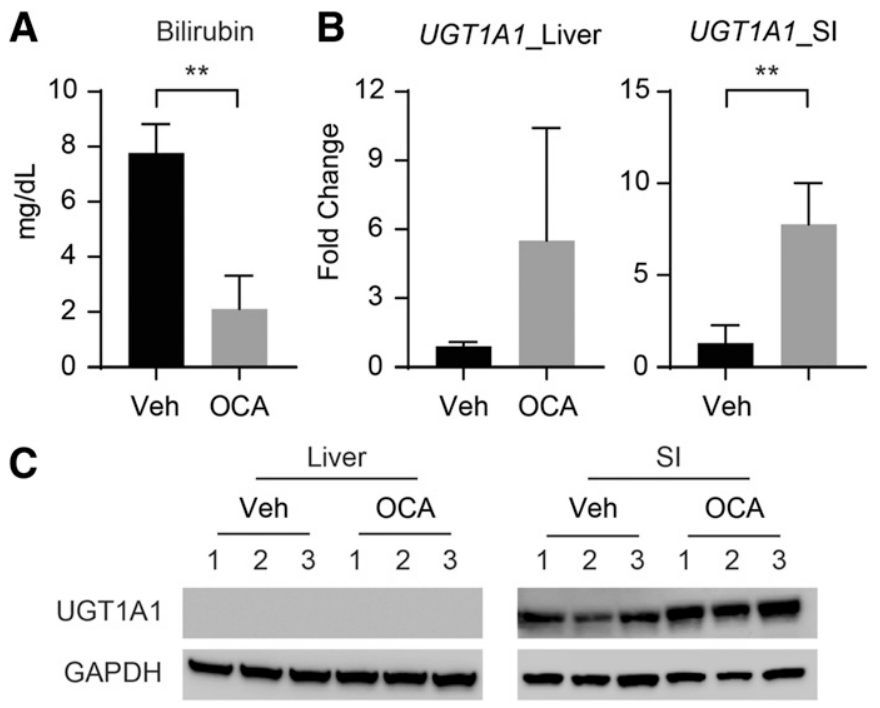

Fig. 1. Induction of UGTIAl by OCA in neonatal $h U G T 1$ mice. The 10-day-old neonatal $h U G T 1$ mice were treated with $50 \mathrm{mg} / \mathrm{kg}$ of OCA (divided in two doses of $25 \mathrm{mg} / \mathrm{kg}$ ) or vehicle by oral administration for 5 consecutive days. (A) After the treatment, TSB levels were measured. (B) Liver and SI were used for gene and protein analysis. UGT1A1 gene expression was determined by RT-qPCR and expressed as fold induction. (C) Western blots were performed from liver and SI tissue to examine UGT1A1 expression. The bands have been cropped, and the fulllength blots are in Supplemental Material. Values are means \pm S.D. $(n \geq 3)$. Statistically significant differences between vehicle (Veh) and OCA are indicated by asterisks (Student's $t$ test: $* * P<0.01$ ). RT-qPCR, reverse-transcription quantitative polymerase chain reaction.

pathways, was not affected by the treatment (Fig. 2B). This result suggests that OCA affects both classic and alternate pathways of bile acid metabolism.

The Role of CAR in Intestinal UGT1A1. Among the target genes of the nuclear receptors or environmental sensors known to regulate UGT1A1, intestinal Cyp2b10, a target gene of activated CAR, was substantially induced by OCA treatment. Along with gene expression, Western blot analysis confirmed strong induction of CYP2B10 in intestines (Fig. 3A). This result indicates a possible role for activated CAR leading to induction of intestinal UGT1A1. A slight increase was also observed for the PXR target gene Cyp3a11, with no statistical differences to nuclear factor erythroid-2-related factor $2, \operatorname{PPAR} \alpha$, and AhR target genes (NADPH dehydrogenase quinone 1, Cyp4a10, and Cypla1, respectively) (Supplemental Fig. 2).

To examine the possibility that activated CAR is inducing UGT1A1, 10-day-old $h U G T 1 / \mathrm{Car}^{-1-}$ mice were treated by gavage with OCA, and analysis of gene expression and protein levels was monitored in both liver and SI. TSB levels were not reduced when $h U G T 1 / \mathrm{Car}^{-1-}$ mice were treated with OCA when compared with $h U G T 1 / \mathrm{Car}^{-1-}$ neonatal mice that received only vehicle (Fig. 3B). Also, there was no induction of intestinal UGT1A1 and CYP2B10 in OCA-treated $h U G T 1 / \mathrm{Car}^{-/-}$ mice, confirming that $\mathrm{CAR}$ plays an important role in inducing intestinal UGT1A1 in mice treated with OCA (Fig. 3C). With this result, the possibility exists that $\mathrm{CAR}$ and FXR are working synergistically to induce UGT1A1 and CYP2B10, a property that might impact directly on FXR activation. When we examined FXR target genes in intestines, Shp and Fgfl5 were induced in hUGTl and hUGTl/ $\mathrm{Car}^{-1-}$ mice treated with OCA (Fig. 4A), whereas in liver, the Cyp7al and Shp gene, which is blocked by FGF15, was inhibited in hUGT1 and hUGT1/Car ${ }^{-/-}$ mice, demonstrating that CAR is not involved in cellular crosstalk with FXR activation by OCA (Fig. 4B).

OCA Accelerates Intestinal Maturation in a CAR-Dependent Fashion. Since OCA is not a CAR ligand, we elected to examine 

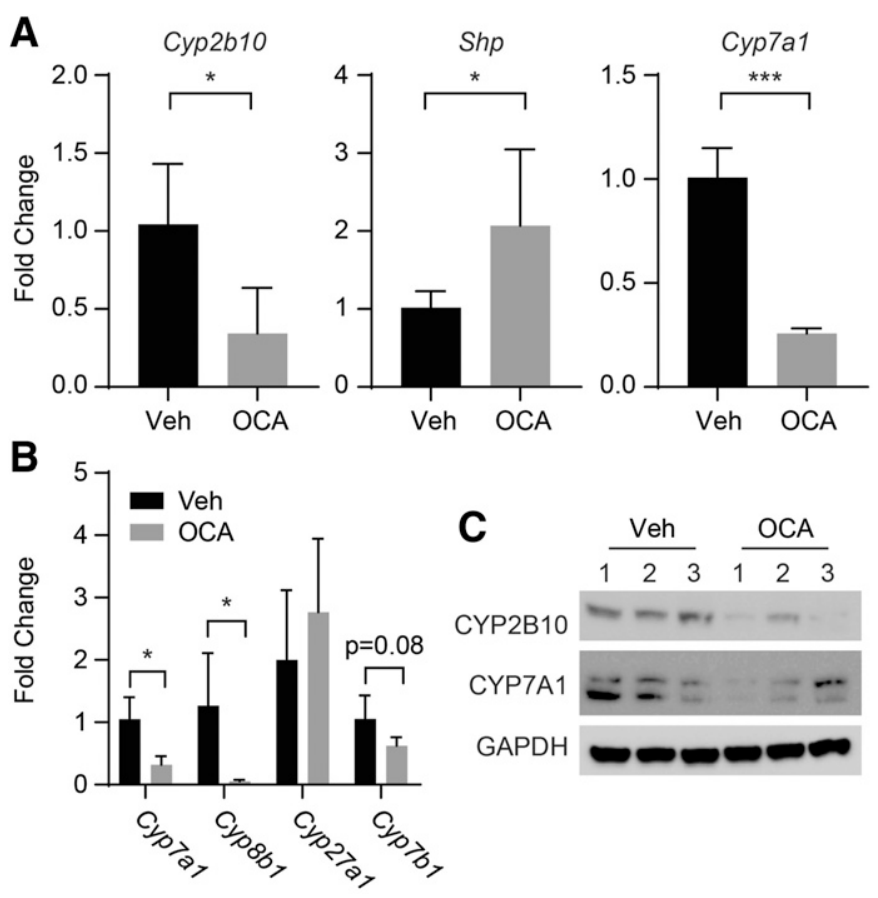

Fig. 2. Oral OCA effect in liver of $h U G T 1$ mice. Litters were bred to produce hUGT1 mice, and 10-day-old neonatal mice were treated with OCA or vehicle on day 10, followed by tissue preparation after treatment. (A) RNA was isolated from liver and used to conduct RT-qPCR for Cyp2b10, Shp, and Cyp7al genes.Supplemental Material (B) Total RNA was prepared from liver, and RT-qPCR was performed to measure expression of Cyp7a1, Cyp8b1, Cyp27a1, and Cyp7b1. (C) Western blots were performed using antibodies toward mouse CYP2B10 and mouse CYP7A1. The bands have been cropped, and the full-length blots are in Supplemental Material. Values are means \pm S.D. $(n \geq 3)$. Statistically significant differences between vehicle (Veh) and OCA are indicated by asterisks (Student's $t$ test: $* P<0.05, * * * P<0.001)$. RT-qPCR, reverse-transcription quantitative polymerase chain reaction.

selective intestinal epithelial cell gene markers that are linked to epithelial cell maturation and proliferation. Several lines of evidence indicate that accelerated intestinal epithelial cell maturation has a direct impact on induction of the intestinal UGT1A1 gene in $h U G T 1$ mice (Chen et al., 2017). When the intestinal nuclear receptor corepressor 1 (Ncorl) gene was targeted for deletion in $h U G T 1$ mice, hyperbilirubinemia in neonatal $h U G T 1 / N C o R l^{\Delta S I}$ mice was completely diminished (Chen et al., 2017). When we investigated the underlying mechanism leading to induction of intestinal UGT1A1 and reduction in serum bilirubin, it was discovered that NCoR1 deletion induced intestinal epithelial cell (IEC) maturation, as determined by accelerated IEC proliferation along with IEC maturation marker genes that are regulated during the maturation process (Chen et al., 2017; Yoda et al., 2017). Sucrase-isomaltase (SIS) consists of two subunits, sucrase and isomaltase, that form a dimer that is anchored on the brush border to generate glucosidase activity (Wang et al., 2001). SIS only exists in differentiated duodenal and jejunal enterocytes and has been a marker of enterocyte maturation, and it is generated as a single polymer that is cleaved, forming the two subunits (Hauri et al., 1985). After oral OCA treatment, induction of the Sis gene expression was significantly induced along with induction of SIS protein, as demonstrated by Western blot analysis (Fig. 5A). Additional markers of intestinal maturation are alkaline phosphatase 3 (AKP3) and jejunal-specific keratin 20 (KRT20). Both intestinal Akp3 and Krt20 genes were induced after OCA treatment (Fig. 5A). During maturation, other proteins are downregulated, such as NADPH oxidase 4 (NOX4), a protein that is downregulated at the suckling-to-weaning transition (Mochizuki et al., 2009; Muncan et al., 2011). After OCA treatment, the Nox4 gene is repressed (Fig. 5A). Combined, these findings demonstrate that OCA is accelerating intestinal tissue maturation, a finding previously demonstrated to have a direct impact on expression of intestinal UGT1A1.

OCA induction of intestinal UGT1A1 is CAR-dependent. To determine whether there is a connection between CAR dependence and the induction of intestinal maturation, the maturation marker genes were measured in $h U G T 1 / \mathrm{Car}^{-1-}$ neonatal mice after oral administration of OCA. Unlike the induction patterns of these genes in $h U G T 1$ mice, induction of Sis, Akp3, and Krt2O or the downregulation of Nox4 were not regulated in OCA-treated $h U G T 1 / \mathrm{Car}^{-1-}$ neonatal mice (Fig. 5B). These findings indicate that OCA induction of intestinal maturation during the neonatal period is CAR-dependent.

Treatment of OCA in Organoids. Sato et al. (2009) established long-term culture conditions in which one single Leucine-rich repeatcontaining receptor positive stem cell embedded in matrix gel could independently generate villuslike epithelial organoids containing all differentiated IECs that are reminiscent to the normal gut (Sato and Clevers, 2013). We have shown previously that activation of PXR, $\operatorname{PPAR} \alpha, \mathrm{LXR}, \mathrm{AhR}$, and CAR with selective ligands results in downstream targeted gene expression, confirming that the family of nuclear receptors is functional in intestinal crypt organoids ( $\mathrm{Lu}$ et al., 2017). To determine whether OCA is capable of impacting intestinal crypt organoid differentiation in a pattern similar to that observed with intestinal tissue from treated $h U G T 1$ mice, we treated crypt organoid cultures isolated from 12-day-old hUGT1 mice with OCA at 50 and
A

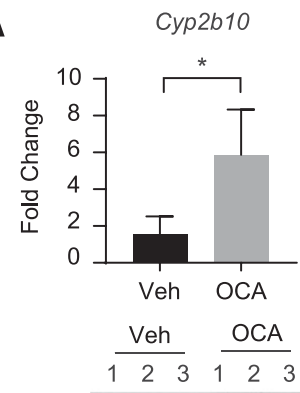

CYP2B10

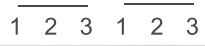
GAPDH
B

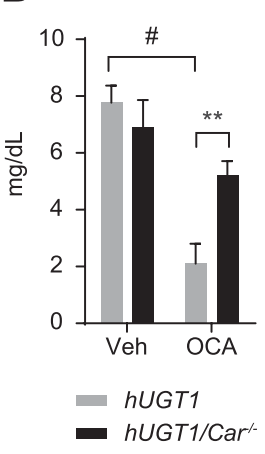

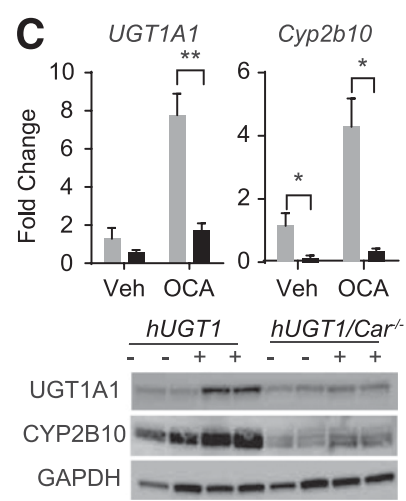

Fig. 3. Oral OCA treatment and effects in intestines of $h U G T 1$ and hUGTI/ $\mathrm{Car}^{-1-}$ mice. Litters were bred to produce hUGTl and $h U G T 1 / \mathrm{Car}^{-1-}$ mice, and 10-day-old neonatal mice were treated with OCA or vehicle on day 10 , followed by tissue preparation after treatment. (A) Intestinal $h U G T 1$ Cyp2b10 was analyzed by RT-qPCR and Western Blot analysis. (B) After treatment, serum bilirubin levels were determined. (C) Intestinal UGTIAl and Cyp 2 b10 gene expression was examined by RT-qPCR from OCA-treated hUGT1 and $h U G T 1 / \mathrm{Car}^{-1-}$ mice. From those same tissues, total cell extracts were prepared for Western blot analysis using anti-UGT1A1 and anti-CYP2B10 antibodies. The bands have been cropped, and the full-length blots are in Supplemental Material. Values are means \pm S.D. $(n \geq 3)$. Statistically significant differences between vehicle (Veh) and OCA are indicated by asterisks in A (Student's $t$ test: ${ }^{*} P<0.05$ ) and statistical differences between groups are indicated by asterisks (Student's $t$ test: $* P<$ $0.05 ; * * \mathrm{P}<0.01)$ and between treatments are indicated as \# in B and C.RT-qPCR, reverse-transcription quantitative polymerase chain reaction. 
A

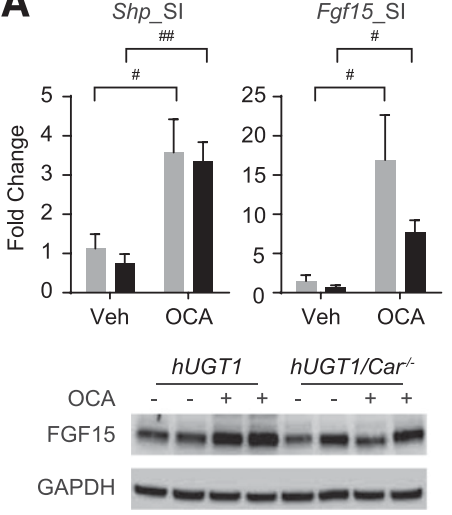

B

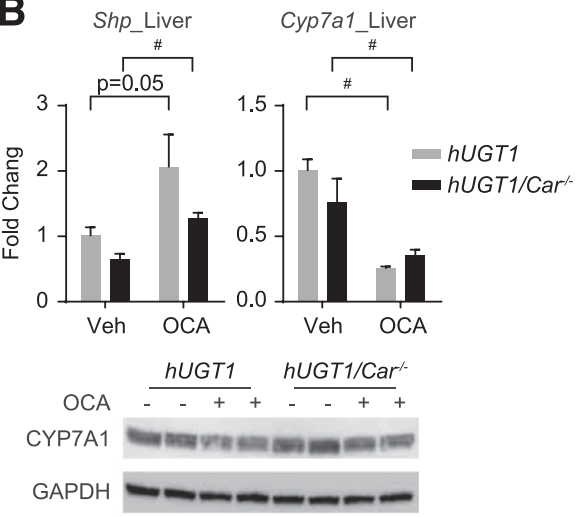

Fig. 4. OCA treatment promotes FXR target gene induction. (A) Total RNA and cellular extracts were prepared from small intestine and liver of $h U G T 1$ and $h U G T 1 / \mathrm{Car}^{-/-}$neonatal mice. (A) Shp and Fgf15 gene expression from small intestines was determined by RTqPCR. Cellular extracts were prepared for Western blot analysis to examine FGF15 protein expression. (B) Liver Shp and Cyp7a1 gene expression was determined by RT-qPCR, and Cyp7a1 protein expression was examined by Western blot analysis. The bands have been cropped, and the full-length blots are in Supplemental Material. Values are means \pm S.D. $(n \geq 3)$. Statistically significant differences between vehicle (Veh) and OCA are indicated by asterisks (Student's $t$ test: ${ }^{\#} P<0.05$; ${ }^{\# \#} P<0.01$; between treatments). RT-qPCR, reverse-transcription quantitative polymerase chain reaction.
$100 \mu \mathrm{m}$ for 24 hours. The treatment of the organoids at both concentrations showed a robust induction of Shp and Fgfl5 gene expression (Fig. 6A) with a significant increase in FGF15 protein levels (Fig. 6E), indicating that OCA treatment leads to FXR activation. Only at the higher concentration were UGT1A1 gene expression (Fig. 6B) and protein levels (Fig. 6E) slightly induced, with the values being statistically significant. However, unlike the dramatic induction of intestinal Cyp $2 b 10$ gene and protein expression by OCA in $h U G T 1$ mice, there was a reduction or inhibition of $C y p 2 b 10$ gene expression in organoid cultures. As we demonstrate in Fig. 5, when neonatal hUGT1 mice were treated with OCA, intestinal maturation marker genes Sis and $A k p 3$ were induced, consistent with IEC differentiation. However, when crypt organoids were treated with OCA, there was a significant reduction in expression of these maturation marker genes (Fig. 6D). To validate that the organoids were capable of differentiation, they were exposed to DAPT, a known $\gamma$-secretase and Notch signaling inhibitor known to drive IEC differentiation (Ogaki et al., 2013). DAPT had no impact on
FXR target genes (Fig. 6A) but led to significant induction of the UGT1A1 and Cyp2b10 genes (Fig. 6, B and C), confirming that differentiation is sufficient to drive induction of intestinal UGTIAl gene expression. However, OCA treatment had no impact on crypt organoid differentiation (Fig. 6D). This interesting finding indicates that the actions of OCA on CAR-dependent induction of intestinal UGT1A1 and CYP2B10 in neonatal hUGT1 mice engages important cellular mechanisms in vivo linked to intestinal maturation that are absent when these experiments are conducted in three-dimensional intestinal organoid cultures.

\section{Discussion}

Severe neonatal hyperbilirubinemia is a syndrome that affects approximately 1 million children every year, especially in low- and middle-income countries (Olusanya et al., 2015). Many of the cases can be successfully treated with phototherapy to lower serum bilirubin
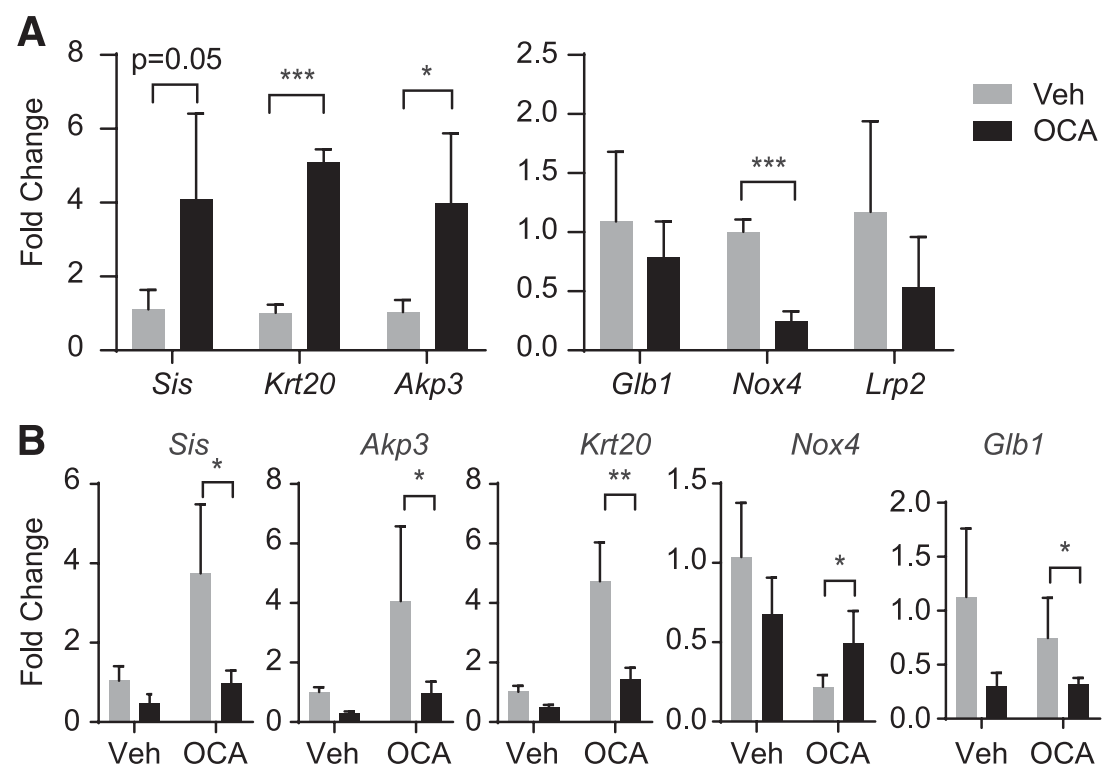

Glb1

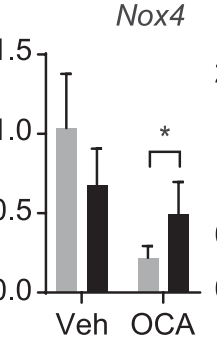

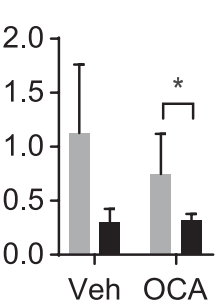

Fig. 5. OCA treatment promotes intestinal maturation. (A) After OCA treatment, total RNA was prepared from small intestine of hUGT1 neonatal mice. Sis, Krt20, Akp3, Glb1, Nox4, and $\operatorname{Lrp} 2$ gene expression was determined by RT-qPCR. (B) After OCA treatment to neonatal $h U G T 1$ and $h U G T 1 / \mathrm{Car}^{-1-}$ mice, small intestine RNA was used to examine Sis, Akp3, Krt20, Nox4, and Glb1 gene expression by RT-qPCR. From total cell extracts, Western blot analysis was performed using antibodies toward mouse SIS. Values are means \pm S.D. $(n \geq 3)$. Statistically significant differences between vehicle (Veh) and OCA are indicated by asterisks (Student's $t$ test: $* P<0.05 ; * * P<0.01 ; * * * P<0.001)$. Nox 4 , NADPH Oxidase 4: Glb1, galactosidase $\beta 1$; Lrp2, low-density lipoprotein-related protein 2; M, mature; P, precursor; RT-qPCR, reverse-transcription quantitative polymerase chain reaction.

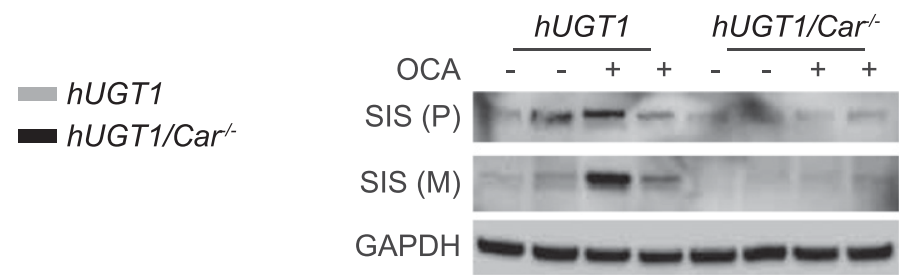



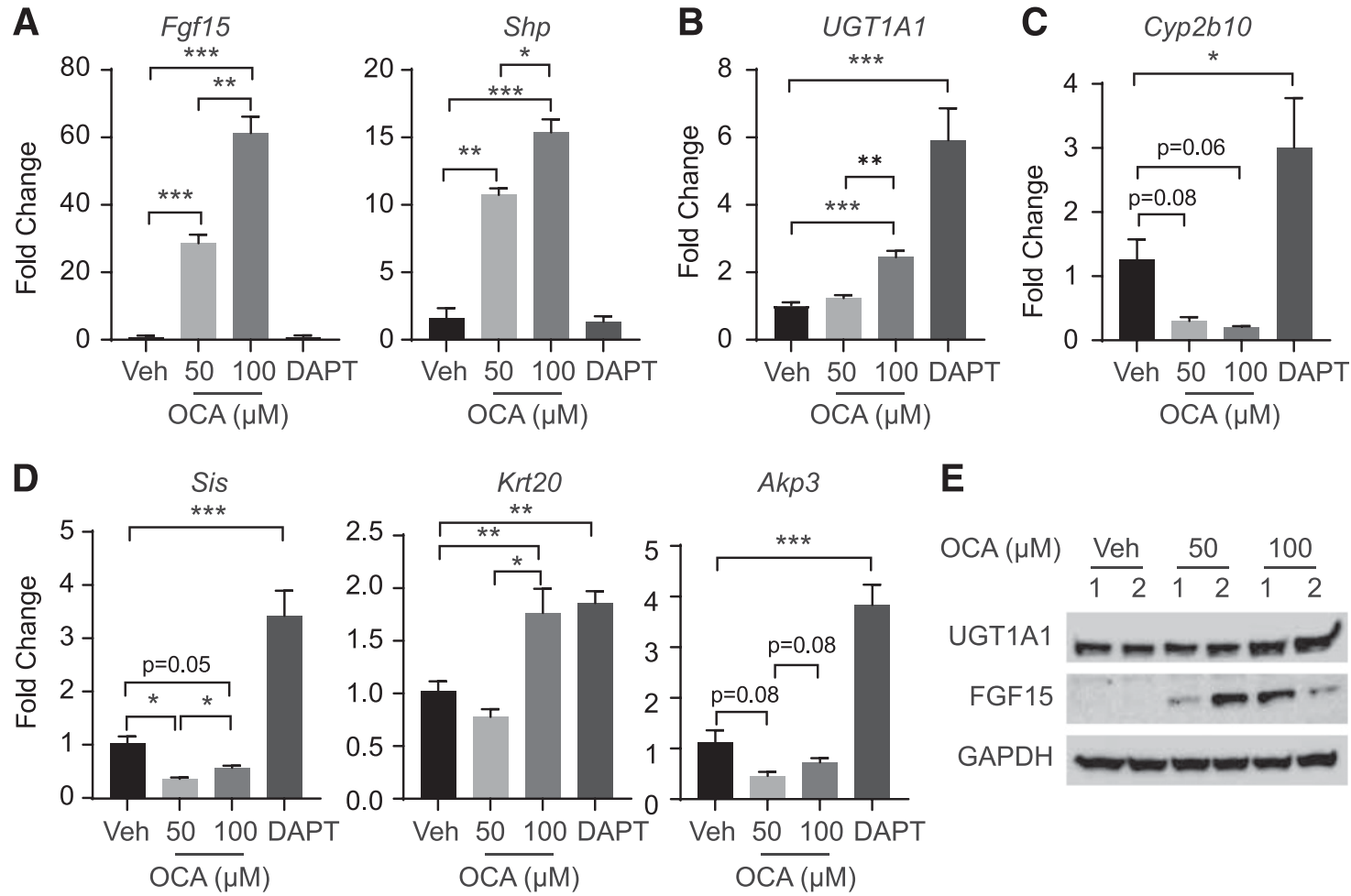

$\mathbf{E}$

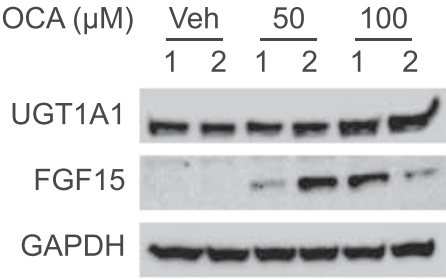

Fig. 6. Treatment of intestinal organoids with OCA. Intestinal organoids cultured from neonatal $h U G T 1$ intestinal tissue were treated with OCA and DAPT for 24 hours. (A) Gene expression of FXR target genes Fgf15 and Shp by RT-qPCR. (B) UGT1A1 gene expression and (C) Cy2b10 gene expression. (D) Gene expression analysis of maturation marker genes Sis, Akp3, and Krt2O. (E) Western blot analysis of UGT1A1 and FGF15 after vehicle (Veh) or OCA treatment. The bands have been cropped, and the full-length blots are in Supplemental Material. Values are means \pm S.D. $(n \geq 3)$. Statistically significant differences between Veh and OCA are indicated by asterisks (Student's $t$ test: $* P<0.05$; $* * P<0.01 ; * * * P<0.001)$. RT-qPCR, reverse-transcription quantitative polymerase chain reaction.

levels, but in more severe situations, additional invasive methods are necessary to decrease bilirubin levels (Mitra and Rennie, 2017). OCA is a semisynthetic derivative of the primary human bile acid chenodeoxycholic acid, the natural agonist of the FXR with anticholestatic and hepatoprotective properties (Pellicciari et al., 2002). This compound has been used to treat patients with type 2 diabetes and nonalcoholic fatty liver disease (Mudaliar et al., 2013). Patients that received 25 or $50 \mathrm{mg}$ of OCA for 6 weeks presented more sensibility to insulin and a reduction in inflammation and fibrosis biomarkers in liver (Mudaliar et al., 2013). In addition to being a drug that promotes benefits for patients with gastrointestinal diseases, we demonstrate that OCA is an effective alternative for the treatment of neonatal hyperbilirubinemia.

According to our RT-qPCR data, both bile acid pathways, classic and alternate, are downregulated in OCA-treated neonatal mice, suggesting that these mice are producing less bile acid. This is consistent with other studies that demonstrate that mice treated with OCA present a reduction of endogenous, primary, and secondary bile acids (Friedman et al., 2018). The suppression of endogenous bile acid by OCA leads to a change in the composition of the gut microbiota (Friedman et al., 2018). Another study has confirmed that the administration of OCA increases the abundance of Blautia in the gut, which is an important bacterium linked to the metabolism of taurine-bound bile acid. This bile acid is abundant when mice are challenged with a high-fat diet, and OCA has been shown to have a protective effect on Nonalcoholic fatty liver disease, a mechanism linked to the gut microbiome (Zhang et al., 2019).

The central function of FXR is to regulate the homeostasis of bile acids (Evans and Mangelsdorf, 2014). The crosstalk between small intestine and liver through the FXR-FGF15/19 axis regulates the production of bile acids from cholesterol (Sinal et al., 2000). However, there is no evidence that FXR has a role in UGT1A1 induction. This was recently brought into question when it was shown that OCA treatment in adult patients with primary biliary cholangitis had the secondary effect of reducing serum bilirubin levels (Parés et al., 2020). Since bilirubin is selectively eliminated through UGT1A1-dependent glucuronidation, this clinical finding suggested that OCA was inducing UGT1A1 through an FXR-dependent mechanism. To examine this possibility, OCA was administered to neonatal $h U G T 1$ mice, and measurement of serum bilirubin, along with intestinal and liver UGT1A1, was performed. It was confirmed that OCA treatment had a direct impact on activation of FXR, with resultant target genes in both the intestines and liver induced. OCA treatment led to a dramatic reduction in serum bilirubin, with significant induction of the intestinal UGTIA1 gene. Although FXR target genes in the liver were induced, confirming that OCA had a direct impact on liver function, hepatic UGT1A1 was not induced. This was unusual, since we have proven previously that activation of additional liver nuclear receptors such as PXR (Chen et al., 2012), CAR (Yoda et al., 2017), $\operatorname{PPAR} \alpha$ (Senekeo-Effenberger et al., 2007), and LXR $\alpha$ (Hansmann et al., 2020) is capable of inducing liver UGT1A1. Thus, the inability of activated FXR in liver to induce UGT1A1 led us to speculate that OCAinitiated induction of intestinal UGT1A1 was occurring through an independent mechanism not directly linked to FXR activation.

The induction of intestinal UGT1A1 by OCA catalyzes the metabolism of serum bilirubin, similar to what we have shown when intestinal UGT1A1 is induced after oral administration of other agents, such as cadmium (Liu et al., 2016; Paszek and Tukey, 2020), arsenic (Liu et al., 2016), isothiocyanates (Yoda et al., 2017; Paszek and Tukey, 2020), and formula (Fujiwara et al., 2012). Induction of intestinal UGT1A1 is sufficient, in the absence of any regulation of liver UGT1A1, to metabolize and clear serum bilirubin. To examine the underlying mechanism of OCA-induced intestinal UGT1A1, we examined a family 


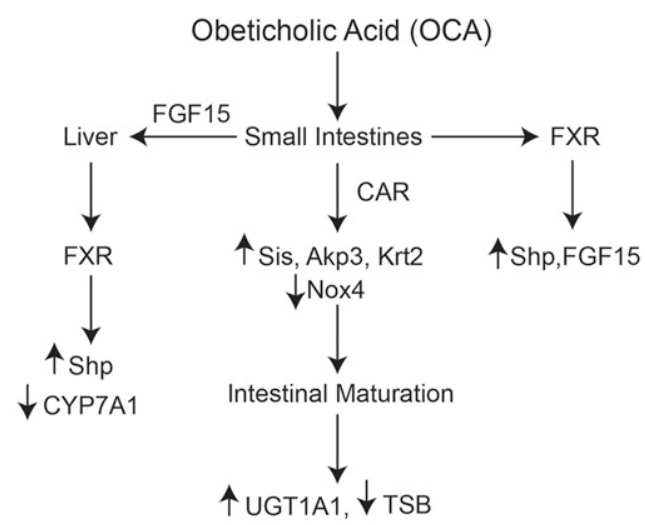

Fig. 7. Pathways involved with OCA treatment. Administration of OCA activates intestinal CAR and FXR and their respective target genes. In small intestines, FXR activation leads to induction of FGF15 and Shp, whereas OCA is capable of inducing liver FXR target genes such as Shp gene expression. Induction of FGF15 results in inhibition of liver Cyp7al gene expression. After oral OCA treatment, CAR becomes activated, leading to an increase in intestinal epithelial cell maturation, as displayed by activation of specific marker genes. Activation of intestinal maturation results in induction of intestinal UGT1A1 that drives the metabolism of serum bilirubin.

of target genes that can be induced by activated nuclear receptors. From this initial screen, intestinal CYP2B10 was prominently induced. In addition, induction of CYP2B10 by OCA only occurred in intestinal tissue, indicating the actions of OCA and induction of CYP2B10 are restricted to intestinal tissue, similar to what is observed for induction of intestinal UGT1A1. Both the human UGT1A1 and mouse Cyp2b10 genes can be induced by activated CAR. To examine the possible connection between OCA-elicited CAR activation and induction of UGT1A1, OCA was administered to neonatal $h U G T 1 / \mathrm{Car}^{-1-}$ mice, followed by measurement of serum bilirubin and expression of UGT1A1 and CYP2B10. The absence of CAR function in $h U G T 1$ mice resulted in limited clearance of serum bilirubin by OCA treatment and no induction of intestinal UGT1A1 and CYP2B10, confirming that the underlying mechanism of OCA induction of intestinal UGT1A1 is CAR-dependent.

There is no evidence that OCA is a direct ligand for CAR, thus indicating that the induction of intestinal UGT1A1 through CAR dependence is occurring indirectly yet in an intestinal-specific manner. One of the key functions of the intestinal tract is to continually renew IECs (Wang et al., 2017). IEC maturation stems from deep intestinal crypt cells proliferating and differentiating into the absorptive and secretory cell lineages that constitute the intestinal villi (Yeung et al., 2011; De Mey and Freund, 2013). The maturation process is tightly controlled through transcriptional repressive actions that include NCoR1, and if these repressive states are interrupted or released, accelerated proliferation and differentiation occurs (Mottis et al., 2013; Jo et al., 2015; Mennillo et al., 2020). We have currently established that the oral treatment of neonatal $h U G T 1$ mice with isothiocyanates induces hepatic UGT1A1 through a CAR-dependent mechanism that is directly linked to the development of hepatic oxidative stress (Yoda et al., 2017). However, this mechanism is not associated with CAR activation in either liver or intestines after OCA treatment. When we examined a series of selective intestinal maturation markers that are either induced or inhibited upon proliferation and differentiation, OCA led to induced neonatal maturation, a process that leads to induction of intestinal UGT1A1. With OCA inducing intestinal UGT1A1 in a CAR-dependent manner, it was demonstrated that accelerated IEC maturation after OCA treatment is also highly dependent on CAR, since IEC maturation or induction of UGT1A1 does not occur in hUGT1/Car ${ }^{-1-}$ mice.
With our findings, we have developed a mechanistic model to explain the actions of OCA toward induction of intestinal UGT1A1 in $h U G T 1$ mice (Fig. 7) As a potent FXR agonist, OCA activates FXR in both liver and intestinal tissue. This was confirmed by induction of FXR target genes, such as Shp, in both tissues, whereas FGF15 produced in the intestines blocks transcriptional activation of the Cyp7al gene in liver. Although FXR is activated in liver and intestines, OCA does not lead to the induction of liver UGT1A1, confirming that FXR, as well as other potential nuclear receptors known to induce UGT1A1, such PXR, $\operatorname{PPAR} \alpha, \mathrm{LXR} \alpha$, and CAR, are not activated in this tissue. However, intestinal UGT1A1 is significantly induced in the small intestine after OCA treatment. Associated with this induction is an absolute dependence on CAR, which is also tightly linked to IEC maturation. Since CAR is associated with UGT1A1 induction in small intestines and not liver, additional tissue-specific factors associated with the intestines must be playing an important role in the activation of CAR, which also controls premature IEC maturation (Fig. 7).

\section{Authorship Contributions}

Participated in research design: Weber, van der Schoor, Jonker, Chen, Tukey.

Conducted experiments: Weber, Mennillo, Yang.

Wrote or contributed to the writing of the manuscript: Weber, Tukey.

\section{References}

Barateiro A, Chen S, Yueh MF, Fernandes A, Domingues HS, Relvas J, Barbier O, Nguyen N, Tukey RH, and Brites D (2016) Reduced myelination and increased glia reactivity resulting from severe neonatal hyperbilirubinemia. Mol Pharmacol 89:84-93.

Bhutani VK and Johnson-Hamerman L (2015) The clinical syndrome of bilirubin-induced neurologic dysfunction. Semin Fetal Neonatal Med 20:6-13.

Bhutani VK, Zipursky A, Blencowe H, Khanna R, Sgro M, Ebbesen F, Bell J, Mori R, Slusher TM, Fahmy N, et al. (2013) Neonatal hyperbilirubinemia and Rhesus disease of the newborn: incidence and impairment estimates for 2010 at regional and global levels. Pediatr Res $\mathbf{7 4}$ (Suppl 1):86-100.

Chen S, Lu W, Yueh MF, Rettenmeier E, Liu M, Paszek M, Auwerx J, Yu RT, Evans RM, Wang $\mathrm{K}$, et al. (2017) Intestinal NCoR1, a regulator of epithelial cell maturation, controls neonatal hyperbilirubinemia [published correction appears in Proc Natl Acad Sci USA (2017) 114: E4115]. Proc Natl Acad Sci USA 114:E1432-E1440.

Chen S and Tukey RH (2018) Humanized UGT1 mice, regulation of UGT1A1, and the role of the intestinal tract in neonatal hyperbilirubinemia and breast milk-induced jaundice. Drug Metab Dispos 46:1745-1755.

Chen S, Yueh MF, Evans RM, and Tukey RH (2012) Pregnane-x-receptor controls hepatic glucuronidation during pregnancy and neonatal development in humanized UGT1 mice. Hepatology 56:658-667.

Cunningham AD, Hwang S, and Mochly-Rosen D (2016) Glucose-6-Phosphate dehydrogenase deficiency and the need for a novel treatment to prevent kernicterus. Clin Perinatol 43:341-354. De Mey JR and Freund JN (2013) Understanding epithelial homeostasis in the intestine: an old battlefield of ideas, recent breakthroughs and remaining controversies. Tissue Barriers 1:e24965. Evans RM and Mangelsdorf DJ (2014) Nuclear receptors, RXR, and the big bang. Cell 157: 255-266.

Friedman ES, Li Y, Shen TD, Jiang J, Chau L, Adorini L, Babakhani F, Edwards J, Shapiro D, Zhao C, et al. (2018) FXR-dependent modulation of the human small intestinal microbiome by the bile acid derivative obeticholic acid. Gastroenterology 155:1741-1752.e5.

Fujiwara R, Chen S, Karin M, and Tukey RH (2012) Reduced expression of UGT1A1 in intestines of humanized UGT1 mice via inactivation of NF- $\mathrm{KB}$ leads to hyperbilirubinemia. Gastroenterology 142:109-118.e2.

Fujiwara R, Nguyen N, Chen S, and Tukey RH (2010) Developmental hyperbilirubinemia and CNS toxicity in mice humanized with the UDP glucuronosyltransferase 1 (UGTI) locus. Proc Natl Acad Sci USA 107:5024-5029.

Hansmann E, Mennillo E, Yoda E, Verreault M, Barbier O, Chen S, and Tukey RH (2020) Differential role of Liver X Receptor (LXR) $\alpha$ and (LXR) $\beta$ in the regulation of UDPglucuronosyltransferase 1A1 in humanized UGT1 mice. Drug Metab Dispos 48:255-263.

Hauri HP, Roth J, Sterchi EE, and Lentze MJ (1985) Transport to cell surface of intestinal sucraseisomaltase is blocked in the Golgi apparatus in a patient with congenital sucrase-isomaltase deficiency. Proc Natl Acad Sci USA 82:4423-4427.

Jo YS, Ryu D, Maida A, Wang X, Evans RM, Schoonjans K, and Auwerx J (2015) Phosphorylation of the nuclear receptor corepressor 1 by protein kinase B switches its corepressor targets in the liver in mice. Hepatology 62:1606-1618.

Kaplan M, Bromiker R, and Hammerman C (2011) Severe neonatal hyperbilirubinemia and kernicterus: are these still problems in the third millennium? Neonatology 100:354-362.

Kliewer SA and Mangelsdorf DJ (2015) Bile acids as hormones: the FXR-FGF15/19 pathway. Dig Dis 33:327-331.

Liu M, Chen S, Yueh MF, Fujiwara R, Konopnicki C, Hao H, and Tukey RH (2016) Cadmium and arsenic override NF- $\mathrm{B}$ developmental regulation of the intestinal UGT1A1 gene and control of hyperbilirubinemia. Biochem Pharmacol 110-111:37-46.

Lu W, Rettenmeier E, Paszek M, Yueh MF, Tukey RH, Trottier J, Barbier O, and Chen S (2017) Crypt organoid culture as an in vitro model in drug metabolism and cytotoxicity studies. Drug Metab Dispos 45:748-754. 
Maisels MJ (2015) Managing the jaundiced newborn: a persistent challenge. CMAJ 187:335-343 Mennillo E, Yang X, Paszek M, Auwerx J, Benner C, and Chen S (2020) NCoR1 protects mice from dextran sodium sulfate-induced colitis by guarding colonic crypt cells from luminal insult. Cell Mol Gastroenterol Hepatol 10:133-147.

Mitra S and Rennie J (2017) Neonatal jaundice: aetiology, diagnosis and treatment. Br J Hosp Med (Lond) 78:699-704.

Mochizuki K, Yorita S, and Goda T (2009) Gene expression changes in the jejunum of rats during the transient suckling-weaning period. J Nutr Sci Vitaminol (Tokyo) 55:139-148.

Mottis A, Mouchiroud L, and Auwerx J (2013) Emerging roles of the corepressors NCoR1 and SMRT in homeostasis. Genes Dev 27:819-835.

Mudaliar S, Henry RR, Sanyal AJ, Morrow L, Marschall HU, Kipnes M, Adorini L, Sciacca CI, Clopton P, Castelloe E, et al. (2013) Efficacy and safety of the farnesoid X receptor agonist obeticholic acid in patients with type 2 diabetes and nonalcoholic fatty liver disease. Gastroenterology 145:574-582.e1.

Muncan V, Heijmans J, Krasinski SD, Büller NV, Wildenberg ME, Meisner S, Radonjic M, Stapleton KA, Lamers WH, Biemond I, et al. (2011) Blimp1 regulates the transition of neonatal to adult intestinal epithelium. Nat Commun 2:452.

Ogaki S, Shiraki N, Kume K, and Kume S (2013) Wnt and Notch signals guide embryonic stem cell differentiation into the intestinal lineages. Stem Cells 31:1086-1096.

Olusanya BO, Osibanjo FB, and Slusher TM (2015) Risk factors for severe neonatal hyperbilirubinemia in low and middle-income countries: a systematic review and meta-analysis. PLoS One 10:e117229.

Parés A, Shiffman M, Vargas V, Invernizzi P, Malecha ES, Liberman A, MacConell L, and Hirschfield G (2020) Reduction and stabilization of bilirubin with obeticholic acid treatment in patients with primary biliary cholangitis. Liver Int 40:1121-1129.

Paszek M and Tukey RH (2020) NRF2-Independent regulation of intestinal constitutive androstane receptor by the pro-oxidants cadmium and isothiocyanate in hUGT1 mice. Drug Metab Dispos 48:25-30.

Pellicciari R, Fiorucci S, Camaioni E, Clerici C, Costantino G, Maloney PR, Morelli A, Parks DJ, and Willson TM (2002) 6alpha-ethyl-chenodeoxycholic acid (6-ECDCA), a potent and selective FXR agonist endowed with anticholestatic activity. J Med Chem 45:3569-3572.

Sato $\mathrm{T}$ and Clevers H (2013) Growing self-organizing mini-guts from a single intestinal stem cell: mechanism and applications. Science 340:1190-1194.

Sato T, Vries RG, Snippert HJ, van de Wetering M, Barker N, Stange DE, van Es JH, Abo A Kujala P, Peters PJ, et al. (2009) Single Lgr5 stem cells build crypt-villus structures in vitro without a mesenchymal niche. Nature 459:262-265.
Senekeo-Effenberger K, Chen S, Brace-Sinnokrak E, Bonzo JA, Yueh MF, Argikar U, Kaeding J, Trottier J, Remmel RP, Ritter JK, et al. (2007) Expression of the human UGT1 locus in transgenic mice by 4-chloro-6-(2,3-xylidino)-2-pyrimidinylthioacetic acid (WY-14643) and implications on drug metabolism through peroxisome proliferator-activated receptor alpha activation. Drug Metab Dispos 35:419-427.

Sinal CJ, Tohkin M, Miyata M, Ward JM, Lambert G, and Gonzalez FJ (2000) Targeted disruption of the nuclear receptor FXR/BAR impairs bile acid and lipid homeostasis. Cell 102:731-744.

Wang Q, Wang X, Hernandez A, Kim S, and Evers BM (2001) Inhibition of the phosphatidylinositol 3-kinase pathway contributes to HT29 and Caco-2 intestinal cell differentiation. Gas troenterology 120:1381-1392.

Wang Q, Zhou Y, Rychahou P, Fan TW, Lane AN, Weiss HL, and Evers BM (2017) Ketogenesis contributes to intestinal cell differentiation. Cell Death Differ 24:458-468.

Wong RJ and Stevenson DK (2015) Neonatal hemolysis and risk of bilirubin-induced neurologic dysfunction. Semin Fetal Neonatal Med 20:26-30.

Yeung TM, Chia LA, Kosinski CM, and Kuo CJ (2011) Regulation of self-renewal and differentiation by the intestinal stem cell niche. Cell Mol Life Sci 68:2513-2523.

Yoda E, Paszek M, Konopnicki C, Fujiwara R, Chen S, and Tukey RH (2017) Isothiocyanates induce UGT1A1 in humanized UGT1 mice in a CAR dependent fashion that is highly dependen upon oxidative stress. Sci Rep 7:46489.

Yueh MF, Huang YH, Hiller A, Chen S, Nguyen N, and Tukey RH (2003) Involvement of the xenobiotic response element (XRE) in Ah receptor-mediated induction of human UDPglucuronosyltransferase 1A1. J Biol Chem 278:15001-15006.

Yueh MF and Tukey RH (2007) Nrf2-Keap1 signaling pathway regulates human UGT1A1 expression in vitro and in transgenic UGT1 mice. J Biol Chem 282:8749-8758.

Zhang DY, Zhu L, Liu HN, Tseng YJ, Weng SQ, Liu TT, Dong L, and Shen XZ (2019) The protective effect and mechanism of the FXR agonist obeticholic acid via targeting gut microbiota in non-alcoholic fatty liver disease. Drug Des Devel Ther 13:2249-2270.

Address correspondence to: Robert H. Tukey, Department of Pharmacology, University of California, San Diego, 9500 Gilman Dr., La Jolla, CA 92093-0722. E-mail: rtukey@health.ucsd.edu 